\title{
Conceptual representations in goal-directed decision making
}

\author{
Nicholas Shea ANd Kristine KRUg \\ University of Oxford, Oxford, England \\ AND \\ Philippe N. Tobler \\ University of Cambridge, Cambridge, England
}

\begin{abstract}
Emerging evidence suggests that the long-established distinction between habit-based and goal-directed decision-making mechanisms can also be sustained in humans. Although the habit-based system has been extensively studied in humans, the goal-directed system is less well characterized. This review brings to that task the distinction between conceptual and nonconceptual representational mechanisms. Conceptual representations are structured out of semantic constituents (concepts) - the use of which requires an ability to perform some language-like syntactic processing. Decision making - as investigated by neuroscience and psychology - is normally studied in isolation from questions about concepts as studied in philosophy and cognitive psychology. We ask what role concepts play in the "goal-directed" decision-making system. We argue that one fruitful way of studying this system in humans is to investigate the extent to which it deploys conceptual representations.
\end{abstract}

\section{Introduction}

Many animals have at least two systems for producing instrumental behavior: the habit system and the goaldirected system (Dickinson \& Balleine, 2002). These multiple action and learning systems are the scope of modern learning theory (Balleine \& Dickinson, 1998a), which goes beyond previous models (Dickinson 1980). There is growing evidence that the systems responsible for human instrumental behavior and decision making divide along similar lines (Gottfried, O'Doherty, \& Dolan, 2003; Valentin, Dickinson, \& O'Doherty, 2007). However, it is often found that in many domains, human psychology is more sophisticated than that of other animals. This may also be true of the mechanisms of decision making. In particular, the human capacity for parsing and producing the complex syntactic structures of language may well have an impact on how we formulate plans and decide between available options. More specifically, language mechanisms allow humans to have and think with concepts. This is not to say that something like human language is necessary for concept possession but, rather, that thinking with internal representations that mirror the constituent structure of natural language is sufficient for concept possession. The term concept is used for a variety of phenomena. Some authors use it for the capacity to respond in the same way to a variety of different stimuli-an ability that, as we use the term, could be mediated by a nonconceptual representation. Nor is the capacity to make inferences sufficient for concept possession, because inferences may be made by making transitions in thought between nonconceptual representations. By concept, we mean a constituent of a mental representation: In order to possess concepts, a thinker must have internal representations with a semantically constituent structure-for example, the familiar subject-predicate structure with which some individual is picked out and some property predicated of that individual. (For more detail, see Section 3 below.) The commonsensical idea that people often reason with concepts has been vindicated and refined by a long tradition of work in both cognitive psychology (Murphy, 2004) and philosophy (Evans, 1982; Millikan, 2000; Peacocke, 1992). This review applies some of those insights, asking what role concepts have in generating goaldirected human behavior.

The article is structured as follows. We start in Section 2 by characterizing the difference between the habitbased decision-making system and the goal-directed decision-making system. In Section 3, we introduce the distinction between conceptual and nonconceptual representations, as clarified by work in philosophy. In Section 4, we ask whether the goal-directed decision-making system in nonhuman animals makes use of conceptual representations. Finally, in Section 5, we suggest how that particular question can be addressed in humans, arguing that there is preliminary evidence that the operation of the dedicated decision-making system in humans that is 
homologous to the goal-directed system studied in other animals does - in humans at least - make use of conceptual representations.

\section{Habit-Based Versus Goal-Directed Decision Making}

There is substantial evidence that in many animals, there are two independent mechanisms for selecting actions. Modeling work explains how we might benefit from having two relatively independent decision-making mechanisms operating according to different principles, and how different types of decisions should be divided between them according to their respective strengths (Daw, Niv, \& Dayan, 2005). There are different ways in which such a distinction between decision-making systems might be drawn - for instance, according to whether short-term or long-term options have to be considered (McClure, Laibson, Loewenstein, \& Cohen, 2004). We want to concentrate on the distinction between habit-based decision making and goal-directed decision making, as described by Balleine and Dickinson (1998a, 1998b; Dickinson \& Balleine, 1994, 2002), but in a catholic spirit, without seeking to prejudge important issues in the literature about how the distinction is precisely to be drawn.

The habit system has at its origin a strong stimulusresponse association, but has been more recently extended to incorporate instrumental conditioning through associative learning (Adams \& Dickinson, 1981; Dickinson, 1980, pp. 92-93). The habit system selects from available actions on the basis of stored values for actions of those types (Packard \& Knowlton, 2002). The action values approximate the long-term expectation of reward flowing from an action of that type. The reward need not be immediate. If the action is part of a sequence, its action value will reflect the likelihood of reinforcement at the end of the sequence. Action selection does not require any thinking ahead, but it does depend on a considerable history of exploration to build up appropriate action values. Action values in the habit system encode an appreciation that a given action will tend to lead to a reinforcer, but the associative structures controlling performance encode only limited information about the type of reward and how it will arise from the action (Balleine \& Dickinson, 1998a). The goal-directed system, by contrast, has some way of representing how actions lead to outcomes, where types of outcome are represented independently of their reward value. So it carries information to distinguish between different outcomes, such as quenching thirst and satiating hunger, whereas outcomes are treated by the habit system merely as reinforcers, which strengthen sensorymotor associations. Since the goal-directed system codes outcomes dependent on reward value, there is a litmus test for goal-directed decision making. If an outcome is devalued - for example, by feeding an animal to satiety on a particular kind of food or by degrading the actionoutcome contingency - the goal-directed system can immediately switch to a different decision, whereas the habit-based system is condemned to continue to select actions that lead to outcomes that it now finds unrewarding (because they are devalued), until it has acquired consid- erable experience of the failure to obtain reward, enabling the system to adjust its action values and thus its preferred course of action. Encoding of the action-outcome contingency, as evidenced by immediate and high sensitivity to reward devaluation, is the signature of the goal-directed system (Dickinson \& Balleine, 2002).

A distinction between the goal-directed system and the habit system is most frequently drawn empirically, in terms of encoding action-outcome contingency (Dickinson, 1980), but various other contrasts drawn in the literature may turn out to be different ways to conceptualize the same distinction. Computational theories of instrumental learning can be divided into two classes: model-based and model-free methods. (That distinction can also be applied directly to psychological decision-making mechanisms themselves.) The goal-directed system chooses between available actions on the basis of some kind of model of the causal structure intervening between actions and possible outcomes. This model-based decision method contrasts with the model-free procedure used by the habit system. Yet a third way of making the distinction distinguishes the branching tree structure of a sequence of decisions encoded by the goal-direct systems, and it contrasts that tree structure with the cached action values relied on by the habit system. These could be considered as alternative ways to draw the distinction between the two systems that are usually investigated under the labels "habit-based" and "goal-directed." Since much remains to be discovered about the nature of these two systems, it may turn out that the distinction between them can be drawn more precisely, for which the foregoing distinctions are candidates.

The division into two relatively independent systems is supported by differences in the neural structures involved in the two types of decision making. Converging evidence is found in electrophysiological recordings (Jog, Kubota, Connolly, Hillegaart, \& Graybiel, 1999; Pasupathy \& Miller, 2005) and lesion studies (Balleine \& Dickinson, 1998a; Coutureau \& Killcross, 2003; Killcross \& Coutureau, 2003; Ramirez \& Savage, 2007; Yin, Knowlton, $\&$ Balleine, 2004) that lay out different neuronal substrates for habit-based and goal-directed decision making in animals. Similar results supporting the idea of two separate systems are emerging from $\mathrm{fMRI}$ data in humans (McClure et al., 2004; O'Doherty et al., 2004; Tobler, O'Doherty, Dolan, \& Schultz, 2007). In habit-based systems, the main structures implicated are the striatum and the associated dopaminergic circuitry. The results of studies of phasic dopamine responses by extra-cellular recording in the midbrain and by fast cyclic voltammetry in the striatum are consistent with dopamine neurons being part of the neural basis of the habit system, although there is as yet no direct evaluation of the operation of this system after devaluation. Lesion studies do not directly elucidate the functional role of the phasic dopamine response.

Focusing on the habit-based system, normative models of how choices are calculated in habit-based decision making can now be tested in the neural networks that seem to be involved. Temporal difference learning models have been particularly influential; the system learns by making predictions of outcomes from various actions, undergo- 
ing learning when those predictions are in error, with the dopamine system playing the role of broadcasting a global error signal, as called for by the model (Houk, Adams, \& Barto, 1995; Montague, Dayan, \& Sejnowski, 1996; Schultz, Dayan, \& Montague, 1997). Other parameters suggested by temporal difference models have also been found to correlate with activity in various areas of the brain that are plausibly involved in learning and deciding on the basis of cached action values (e.g., whether reward was received, Lau \& Glimcher, 2007; learning rate, Behrens, Woolrich, Walton, \& Rushworth, 2007). Where rewards are probabilistic, animals appear able to select actions that maximize the probability of reward (Sugrue, Corrado, \& Newsome, 2004, 2005). In a two-alternative perceptual decision task, neurons in macaque lateral intraparietal cortex were found to encode probabilistic action values that are actually used to select an action; firing rates reflect the probability that the visual stimuli favor one action over the alternative, and, strikingly, it appears that the firing rate correlates directly with the cumulative sum of the log likelihood ratios associated with the various sequentially presented stimuli until some threshold is reached, at which point the action to be selected has effectively been decided (Yang \& Shadlen, 2007). The habitbased decision-making system also shows up in striatal activations during appetitive and aversive conditioning in humans (O'Doherty, Dayan, Friston, Critchley, \& Dolan, 2003; Seymour et al., 2004).

In comparison with the habit system, the mechanisms of goal-directed decision making in other animals are less well characterized. Even less is known about how human goal-directed decision-making works. Indeed, there may be more than one decision-making system that operates in a goal-directed way (cf. Rustichini, 2008). A range of brain areas have been implicated in goal-directed behavior. Lesion work in rats points to a role of the prefrontal cortex and dorsal caudate (Balleine \& Dickinson, 1998a; Yin, Knowlton, \& Balleine, 2005). An actual neurophysiological representation of immediate and final goals for a path-finding task has been found in the lateral prefrontal cortex of monkeys (Saito, Mushiake, Sakamoto, Itoyama, $\&$ Tanji, 2005). Human and animal research suggests that the amygdala and the orbitofrontal cortex are crucial in guiding goal-directed behavior (Holland \& Gallagher, 2004). In order to understand the particular roles these areas play, we need to probe the nature of goal-directed behavior. Dickinson and Balleine (2002; Balleine \& Dickinson, 1998a) gave three conditions for decision making to count as goal directed: (1) The outcome should be represented when one is performing the action, (2) behavior should be based on knowing the causal structure of the domain of action (the contingency between choice and outcome), and (3) behavioral choices should be made according to the motivational value of the outcomes (quality, quantity, and probability).

Evidence of (1) and (3) is furnished by demonstrating sensitivity to reward devaluation. As well as feeding an animal to satiety on a particular type of food, an outcome can be devalued by inducing illness to create a food-specific aversion (Balleine \& Dickinson, 1998a). In primates, sensitivity to reward devaluation seems to depend on the orbitofrontal cortex (Baxter, Parker, Lindner, Izquierdo, \& Murray, 2000; Critchley \& Rolls, 1996). In humans, activity in the orbitofrontal cortex decreases in parallel with behavioral reward devaluation (Gottfried et al., 2003; Valentin et al., 2007). Evidence of (2) - that animals represent the causal structure of the domain of action-comes from several sources. It has long been known that rats can make good use of the information they gain by freely exploring a maze in the absence of rewards. Without such prior exploration, it takes rats several trials to learn to go directly to a reward placed in a consistent position in the maze. However, rats given prior experience of the maze go directly to the site of the reward on the first occasion after they discover it (Blodgett, 1929). This behavior demonstrates that the actions they select as they move through the maze on the way to the anticipated reward are informed by a grasp of the causal relationship between action and outcome. Habit-based learning would take more trials, with associated false turns, before a direct route to the reward was learned. Choice behavior of macaques with orbitofrontal cortex lesions suggests that they fail to assign credit appropriately to a particular trial; instead, if they fail to receive the expected reward, the values of actions performed across a range of prior trialsnot just for the trial in which the reward expectation was not met - are altered (Walton, Rudebeck, Bannerman, \& Rushworth, 2007). This result suggests that without this element of the goal-directed decision-making system, primates will be impaired in dealing properly with the causal structure of the action domain. Finally, recording from hippocampal place cells in rats shows that before rats follow a path, place cells are activated in a sequence reflecting the prospective path, and that, before rats make a choice about which route through a maze to follow, place cells are activated in turn, first reflecting one option, and then the other (Johnson \& Redish, 2007). This is preliminary evidence that some goal-directed decision making proceeds by thinking through the structure of the problem domain from various alternative action choices to possible outcomes.

The orbitofrontal cortex appears to be a key component of the goal-directed system because of its function of encoding the current values of various possible outcomes as predicted by contexts and cues (Rolls, Sienkiewicz, \& Yaxley, 1989). Depending on the relatedness of behavioral contexts used, the outcome values appear to be represented relative to the context (Tremblay \& Schultz, 1999) or in an absolute fashion (Padoa-Schioppa \& Assad, 2008), with the latter enabling transitivity. Particularly, the medial part of the human orbitofrontal cortex is likely involved in goal-directed action, as suggested by its encoding of the causal effectiveness of actions in procuring reward (Tanaka, Balleine, \& O'Doherty, 2008). The amygdala (Ramirez \& Savage, 2007) and anterior cingulate (Kennerly, Walton, Behrens, Buckley, \& Rushworth, 2006) appear also to be implicated in encoding and updating outcome values. By contrast, the rat prelimbic cortex and the dorsal caudate are involved in learning and encoding the action-outcome associations (Balleine \& Dickin- 
son, 1998b; Yin et al., 2005). Interspecies comparisons are complicated by anatomical differences. On the basis of connectivity, the rat ventral prelimbic cortex appears to be the homologue of the primate medial orbitofrontal cortex network, the dorsal prelimbic cortex is that of the medial prefrontal cortex, and perhaps the frontal pole is that of the dorsolateral prefrontal cortex (Fuster, 2000; Price, 2007). However, on the basis of function, the rat prelimbic cortex appears to be the homologue of the primate dorsolateral prefrontal cortex (see, e.g., Birrell \& Brown, 2000; McGaughy, Ross, \& Eichenbaum, 2008; but cf. Preuss, 1995). In any case, although there are hints for involvement of the human medial orbitofrontal cortex (Gottfried et al., 2003; Tanaka et al., 2008; Valentin et al., 2007), it remains to be seen whether the dorsolateral prefrontal cortex processes goal-directed actions in humans. It has been widely demonstrated that sensitivity to reward devaluation is destroyed by sufficiently sustained training on a fixed trial type, suggesting that such overtrained choices are taken over by the habit system. The dorsolateral prefrontal cortex does not appear to be involved in exercising knowledge of these overlearned rules.

In short, there is strong evidence for the existence of and neural basis for relatively independent habit-based and goal-directed decision-making systems in primates and other mammals. Current indications make it likely that there is a similar distinction in the mechanisms of human decision making. The purpose of this review is to suggest an aspect in which the human goal-directed decision-making system may be peculiar: in processing conceptual representations (for some or all goal-directed decisions). The next section spells out what it means for a representational system to be conceptual.

\section{Conceptual Versus \\ Nonconceptual Representation}

As studied in cognitive psychology, concepts have, broadly, two roles. First, they are the basis on which we classify objects together as being instances of the same type of thing. We apply our concept of BIRD to a range of observed objects and thereby take the objects to have something in common (to share the property of being $a$ bird) (Murphy, 2004). Second, concepts are the basis for inferences; for example, having applied the concept BIRD to an object, we may infer that it can fly (or that it is likely to). Philosophers' understanding of concepts dovetails nicely with these two features but emphasizes a further, more exacting constraint. A concept, fundamentally, is a reusable constituent of thought (Evans, 1982; Peacocke, 1992). Sentences are the model: Just as words are constituents of sentences and can be reused to make many different claims, so concepts are constituents of thoughts and can be reused to think many different things. For example, beliefs are complete thoughts. They can be true or false. I might think there is a dog, or the cat is on the mat. Both complete thoughts are candidates for truth: They can be true or false (the first true, the second false, as it happens). But I can reuse one of the capacities that I exercised in thinking that first thought: I can think that dog is brown, dogs are warm-blooded, or even the dog is on the mat.
All of those thoughts are true, but the constituent I have reused - the concept DOG - is not itself true (or false): It is not a candidate for truth. Such things are concepts, and having them makes human thought peculiarly powerful. Thus, although the capacity to generalize and the capacity to make inferences are both necessary to concept possession, neither is sufficient: An ability to deploy representations with semantically significant constituent structures is also required.

Concepts are so ubiquitous in our mental lives that it can be hard to see how nonconceptual representation is possible. An example may help. Think of one of those restaurants in which you order dishes by numbers. The waiter's order slip will be a list of numbers: "12, 23, 142, 147" (our Friday night Chinese meal for two). In the kitchen, the chef reads the numbers as a list of instructions. The number "142" tells him to prepare one portion of Sichuan beef with rice; "147" means prepare one portion of beef in oyster sauce with noodles. Thus, the symbols on the paper are a series of complete instructions, corresponding to complete thoughts (not ones that can be true or false, like beliefs, but ones that can be satisfied/achieved or unsatisfied/failed, like desires and intentions). But the symbols themselves ("142," "147") have no relevant constituent structure. Nothing in the representation corresponds separately to beef, rice, or noodles. Our world is full of examples of nonconceptual representation. The Union Flag flying from the ramparts of Windsor Castle proudly declares that the Queen is in residence, but the flag has no constituent corresponding to the Queen, or to being in residence. Road signs similarly express complete truth-evaluable claims (There is a junction ahead) or instructions (Do not park here) without having any relevant constituent structure. What makes these all examples of genuine representation, which surely they are, is a more controversial question. It is doubtless something to do with the circumstances in which such signs are found and how we tend to act in response to them. What the examples do show is that there is no bar to a representation that itself has no constituent structure being evaluable as a complete declaration or instruction, or for truth or satisfaction. Although we, acting as theorists, would typically use a sentence with constituent structure to express the representation's truth condition, we are not committed to the representation itself having any relevant constituent structure.

A major advantage of deploying conceptual representations should be immediately apparent. Although this merit is familiar, it is hard to notice that it is in any way extraordinary. Only by comparison with the limitations of nonconceptual representation does the power of a system of conceptual representations become clear. To return to our Chinese meal example, suppose the waiter had instead recorded our order using representations with relevant constituent structures - for example, written English. Then it would be very easy for the chef to see the commonalities between the various things ordered. He could just read off how many portions of beef he would have to get to fill the order, how many portions of rice, and so on. But since the number-based order has no constituents corresponding to 
beef, rice, or noodles, the chef's only way of working this out is much more laborious. That is a key merit of conceptual representation: It facilitates the making of a wider range of inferences. Thus, the second of the two central roles that concepts play is to enable useful inferences.

Let us give two examples of the kind of inferences that are facilitated by a capacity for conceptual representation. Suppose I notice that a particular tree has a particular property. For example, one autumn I might notice that Kett's Oak is golden-brown. This thought has the structure $\mathrm{Fa}$, with a singular term (a) that refers to the particular tree, and a predicate $(\mathrm{F})$ that refers to the property of being golden-brown. Suppose, now, that I perform an action A- a search of my vicinity-and get a rewarding outcome $\mathrm{O}$ in this situation: a tummy full of particularly nourishing acorns. I can now learn to associate the action A with outcome $\mathrm{O}$ in situation Fa. Unless my valuations of outcomes change, this will make me more likely to perform action A if I encounter situation $\mathrm{Fa}$ again. But notice how this little piece of learning can take me further: It can affect what I expect in both other F-situations and other a-situations. When I encounter another golden-brown tree and think $\mathrm{Fb}$, this can also incline me to perform action A, which may also be useful: Other golden-brown things may also drop nutritious nuts. Similarly, if I encounter Kett's Oak again when it is bare $(\mathrm{G})$, not golden-brown (F), I may identify this situation as Ga. Again, because the similarity with the original situation is represented explicitly, what I learned about that situation can be carried over to this one, and Kett's Oak may indeed still have some tasty nuts around it, even after it has dropped its leaves. This intellectual facility compares unfavorably with the limitations of the creature equipped only with nonconceptual representations; let us call it "M." M, too, encounters Kett's Oak in goldenbrown glory and deploys a nonconceptual representation, $\mathrm{R}$, to represent the situation. Suppose M also performs action A (search), leading to outcome O (nourishing nuts). $\mathrm{M}$ can also use its goal-directed decision-making system to learn that in situation $\mathrm{R}$, action A tends to lead to outcome $\mathrm{O}$, and to value action A more highly as a result in such situations, provided he remains motivated to obtain O. But notice that M's capacity to learn from this situation is much more limited. It has learned only that in situation R (Kett's Oak being golden-brown), A leads to O. Since $\mathrm{R}$ has no constituent structure, $\mathrm{M}$ is blind to R's relevant similarity to other potentially rewarding situations, situations that to us - with our conceptual representation of the same situation-are so obviously similar that it is difficult at first to accept that $\mathrm{M}$ cannot notice it.

This little story illustrates the great advantage of conceptual representation in allowing for us to generalize more widely from situations in which we learn. Of course, if $\mathrm{M}$ had a variety of nonconceptual representations, it could make just the same inference: Having conceptual representations makes wide generalization much easier. So, width of generalization is not criterial of conceptual representation. It is no kind of diagnostic test: Systems using nonconceptual representations can generalize too (one example might be the results of Freedman, Riesen- huber, Poggio, \& Miller, 2001). Indeed, the absence of a straightforward one-shot diagnostic test for conceptual representation probably explains the fact that this distinction is underappreciated in the empirical literature (many phenomena that it would be fruitful to study empirically are not easy to study empirically). Systems that deploy conceptual representations will tend-other things being equal - to generalize more widely. How widely they in fact generalize will also depend on what the representations - conceptual or nonconceptual — stand for. We know that some animals, such as rats and pigeons, are capable of some degree of generalization, given sufficient training (McLaren \& Mackintosh 2002; Wright \& Katz 2007), but it is currently unclear whether they use conceptual representations to do so.

In our rather quaint story, the animal $\mathrm{M}$ could represent the learning situation in a more general way, despite being limited to purely nonconceptual resources. It could deploy a different representation, $\mathrm{R}^{*}$, in which it would represent as $\mathrm{R}^{*}$ any situation in which it encountered a golden-brown oak. Now, its learned situation-specific action-outcome association would extend to a wider class of situations. It would have some ability to generalize. Notice, however, that $\mathrm{M}$ still lacks completely one whole dimension of potential generalization that we have, since we deploy a singular term, a, to think about Kett's Oak. So there are in-principle reasons why a system deploying only nonconceptual representations will tend to generalize less widely from experience. And, indeed, experience with training other primates on reward-based tasks confirms this striking difference. A macaque may learn to link a particular sensory input-for instance, white dots moving to the right - to a particular action, like eyes moving to the right, to get a specific reward (Newsome, Britten, \& Movshon, 1989). However, if any part of the task is slightly alteredfor instance, the input dots turn black - then the animal will often have problems transferring the appropriate action to the new situation. A natural explanation is that it has no conceptual representation of moving to the right. In the original situation, it has indeed learned a rule about white dots moving to the right, but it has not learned anything about the property of things moving to the right as such. That distinction can be hard to grasp for us, thinking about these cases as we do through the medium of language. Surely, if the animal has learned a rule for white dots moving to the right, it has thereby also learned a rule for dots moving to the right, or even things moving to the right. Or, if it has not actually learned the rule, drawing the appropriate inference should surely be nearly effortless, should it not? The commonalities are obvious to us. But, equipped only with nonconceptual representations, such commonalities are far from obvious. Since they are not represented explicitly via the constituent structure of representations, they have to be constructed and learned about piecemeal, case by case. So, evidence of limited generalization behavior is evidence that the representations giving rise to the behavior are purely nonconceptual, without being anything like a litmus test. Other evidence is needed about the nature of the mechanisms that are being deployed. In principle, any behavior produced by 
a mechanism of conceptual representations could also be produced by a suitably detailed system of nonconceptual representations. The way the two types of systems would learn is likely to differ; but again, any particular piece of learning data can be accounted for by a nonconceptual system with appropriate prior knowledge or biases. Despite being a deep distinction that makes widespread practical difference, the difference between conceptual and nonconceptual systems of representation is by no means simple to investigate empirically. (In this respect, some readers may be reminded of the science of consciousness; having conscious experiences is a fundamental mental property, and being conscious surely does make a deep functional and practical difference to our lives - yet, it is notoriously resistant to direct empirical study.) Convergent evidence of concept-based decision making may come from several sources. One important source of evidence could be neural. The contribution of a brain region that processes syntactic structures during goal-directed decision making might provide further indication that conceptual representations are in play. We would expect such an area to be activated in tasks when conceptual representations are deployed. Conversely, if it lesioned or was temporarily disrupted through TMS, we would expect deficits in tasks that require conceptual representations. Such a demonstration would require behavioral control for the absence of internal speech occurring specifically during decision making. We suggest that where a system-driving behavior - in humans or other animals - is found to be implicated in the organism's ability to generalize easily and widely from its experience, and where, furthermore, there is converging evidence (neural or behavioral) that the behavioral system relies on some kind of syntactic processing of representations with constituent structure, there is reasonable evidence that the system in question processes conceptual representations.

A second example will illustrate how the difference between conceptual and nonconceptual representations may be particularly relevant to goal-directed decision making. Recall the idea that a central feature of concepts is the way they are deployed in reasoning. Suppose I can identify a number of events: $\mathrm{e}_{1}, \mathrm{e}_{2}$, and $\mathrm{e}_{3}$. Now, using my capacity for conceptual representation, I learn and represent that $e_{1}$ comes before $e_{2}$ in one situation and that $e_{2}$ comes before $e_{3}$ in another. It is easy for me to infer that $e_{1}$ comes before $e_{3}$. Intuitively, it seems that we do make these kinds of inferences when reasoning about what to do-at least sometimes. All goal-directed decision making makes use of the knowledge of the causal structure of the domain in order to infer the connection between some proximal action and some distal outcome via a chain of intermediate actions. Equipped with conceptual representations, we humans may be engaging in a particular subspecies of goal-directed decision making - one that relies on representations with constituent structure in coming to some of its decisions.

\section{Conceptual Representations in the Goal-Directed System in Nonhuman Animals}

We have seen that rats show sensitivity to reward devaluation, which indicates that they are capable of goal- directed decision making. One well-known limitation on their ability to show sensitivity to reward devaluation is illustrative. Rats will reduce the response that leads to a

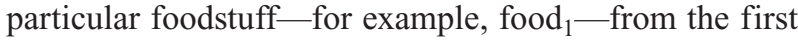
choice they have to make between available options after being sated, but only if they have had the chance to eat food $_{1}$ when sated. They will show sensitivity to the reward devaluation only if they have had a chance to learn the association between the outcome ( food $\left._{1}\right)$ and their drive states (hunger, or lack thereof). Similarly, rats can learn action-outcome associations between pressing a lever and the delivery of food pellets, and between pulling a chain and the delivery of sucrose solution (another foodstuff). Suppose you now make the rat thirsty and offer it a choice between chain and lever (in extinction). Surely it will pull the chain? Yes, but only if it has had previous experience that sucrose solution salves thirst (Balleine \& Dickinson, 1998b; Dickinson \& Balleine, 1994; Dickinson, Balleine, Watt, Gonzalez, \& Boakes, 1995), because only then has it learned that that outcome (the liquid) is rewarding in the drive state of being thirsty. The association between sucrose solution and thirst quenching can be learned from drinking freely available sucrose when thirsty. Shipley and Colwill (1996) suggested that the capacity of rats to generalize might go further. They found in a similar experiment that rats could perform the nondevalued thirst-quenching action even without explicit trials in which they had experienced the sucrose solution when thirsty. However, it is possible that their rats had previously experienced the thirst-quenching properties of the sucrose solution outside the lab (Berridge, 2001). Moreover, the sucrose solution used by Shipley and Colwill (8\%) was less concentrated than the one used by Dickinson and Balleine (20\%). Generalization may therefore have been facilitated by the higher similarity to water for Shipley and Colwill's rats, as compared with those of Dickinson and Balleine. Even Dickinson and Balleine's original claim - that devaluation sensitivity requires experience of sucrose solution's salving thirst - turns out to be too strict; there do seem to be significant limitations on how readily rats can make the generalization from leverpressing when hungry to leverpressing when thirsty. Moreover, the demonstrated sensitivity to reward reevaluation is clear enough: The rat does behave in an entirely new way in the chain/lever situation, as a result of learning something in a different situation in which actions on levers and chains were not involved. Thus, it is clear that a habit-based system that selects actions on the basis of cached action values is a poor candidate to account for the phenomenon.

But, now let us focus on the limitation. We will argue that this type of limitation is important and best explained by the rats' being limited to a system of nonconceptual representations. Although there are ways of explaining the rats' behavior as arising from a purely habit-based system, the standard account has them learning about and using knowledge of the causal structure of the domain. They learn that action (chain pulling) leads to outcome $_{1}$ (sucrose solution), and, if they do so while hungry, they also discover that outcome ${ }_{1}$ sates hunger. However, the rat's knowledge of the association between action $_{1}$ and 
outcome $_{1}$ can be generalized to the situation in which the rat is thirsty only if it somehow knows about the relation between outcome ${ }_{1}$ and quenching thirst (and recall that in order to do so, the rat must have a goal-directed decision-making system and not be restricted to learning that is based on habit/cached action values). If outcome ${ }_{1}$ is represented nonconceptually, that may be achieved by giving the rat experience-outside the apparatus - of the fact that outcome ${ }_{1}$ is rewarding when thirsty. It could also be achieved by representing outcome ${ }_{1}$ with sufficiently general nonconceptual representation (as perhaps occurred in Shipley \& Colwill's [1996] experiment, when the concentration of sucrose in outcome ${ }_{1}$ was only $8 \%$ ). However, if outcome ${ }_{1}$ were represented conceptually, then there would be many more opportunities to make the relevant generalization. Are we humans subject to the same kind of limitation as are the rats, or do we show evidence of our conceptual representations (which we undoubtedly have for other purposes) being deployed within the causal knowledge of the goal-based system? That is, of course, an empirical question that needs much further investigation, and there undoubtedly are situations in which humans do indeed show limitations of just this type. But it is also plausible that we can sometimes overcome them-at least, it is plausible enough so as to commend itself as a hypothesis for detailed empirical investigation. When we receive outcome ${ }_{1}$, our conceptual resources can cut in, allowing us to think, in this cup is a sweet, sticky, clear, liquid. Without doubting that humans, too, have to learn somehow which outcomes are rewarding and in which ways, we can see that this conceptual representation gives us many more chances to make use of existing knowledge. We may have no experience of the relation between sugar solution and thirst quenching, but experience of the connection between liquids and thirst quenching or, indeed, experience of the connection between things in cups and thirst quenching would do. Our behavior is likely to be much less situation bound as a result. Deploying conceptual representations does not obviate the need to learn from experience, it just allows the results of that learning to be leveraged to a much wider range of related situations.

What about other animals? There is a large amount of literature - in both philosophy and comparative psychology - about whether nonhuman animals deploy concepts (see, e.g., Allen \& Hauser, 1996; Bermúdez, 2006; Stich, 1978; and further articles in Hurley \& Nudds, 2006), and whether concept use requires human-like natural language or some language-like syntactic ability that could be possessed by animals not endowed with the full complexities of a language faculty (Bermúdez, 2003; Chater \& Heyes, 1994), with some philosophers arguing that nonhuman animals do not deploy conceptual representations in any of their behavioral systems (e.g., Hurley \& Nudds, 2006). What is new is to ask specifically about the goal-directed decision-making system (as studied in animals, and its homologue in humans), and whether it makes use of conceptual representations. Goal-directed decision making involves reasoning about means and ends and, hence, inference; but as we noted previously, inferences may be performed over nonconceptual representations. The ques- tion is whether the representations between which inferences are made by the goal-directed decision-making system possess constituent structure (e.g., subject-predicate structure). Is there evidence that conceptual representations are deployed by the goal-based decision-making systems found in other animals? In rhesus monkeys, consequences of intended actions for goal-directed behavior are thought to be represented in the lateral prefrontal cortex (Saito et al., 2005; Tanji, Shima, \& Mushiake, 2007). There is also a growing number of studies of rhesus monkeys that describe neuronal signals that appear to represent concepts (Freedman \& Assad, 2006; Shima, Isoda, Mushiake, \& Tanji, 2007). For instance, when monkeys are trained to repeat different temporal sequences of behaviors (AABB, ABAB, CCDD, CDCD), neurons in the prefrontal cortex respond specifically to a category of the temporal patterns (AABB, CCDD) (Shima et al., 2007). Thus, there is little doubt that other animals can generalize from situations that they have encountered to other, similar situations, including making generalizations on the basis of temporal structure. As with any ability to generalize, these could be mediated by representations without constituent structure: nonconceptual representations. The crucial question for our purposes is whether the monkey's behavior transfer depends on representations with constituent structure (more specifically, whether such structured representations are deployed within the goal-directed decision-making system that is our focus here). If a monkey were able to gather a series of pieces of information about an individual — only putting them together for some behavioral purpose at a later date - that would suggest that they have a concept of that individual (although that would be a conservative test: Failure would not be strong evidence against concept use). As we have said, the undoubtedly real and important distinction between representations with and without constituent structure cannot be operationalized into a simple experimental test. Instead, we suggest putting together evidence from generalization to novel contexts with other lines of evidence about the mechanisms involved.

It is undoubtedly impressive that rats and other animals can engage in genuinely goal-directed behavior. But our admiration should not blind us to the significance of the way in which they appear limited. That limitation suggests that they may be restricted to nonconceptual representations, or, if they do have concepts, that their goal-directed decision-making system is encapsulated and does not have access to their conceptual abilities. Of course, it is ultimately more difficult to prove absence than presence of conceptual representations. We need to be cautious, since claims about unique human capacities, as compared with those of other animals, had to be qualified in the past, because of subsequent empirical tests (e.g., episodic memory in scrub jays, Clayton \& Dickinson, 1998; tool use by crows and monkeys, Kenward, Weir, Rutz, \& Kacelnik, 2005; Ottoni, de Resende, \& Izar, 2005; transmission of group-specific types of tool use by chimpanzees, Whiten et al., 2007; asocial perspective taking by chimpanzees and scrub jays, Emery \& Clayton, 2001; Hare, Call, Agnetta, \& Tomasello, 2000; Hare, Call, \& Tomasello, 2001). 
A further caveat is that when entering the lab, humans are usually experienced, whereas animals are naive. With increased experience, animals may indeed show behaviors and solve tasks that naive animals do not perform (see, e.g., Balleine, Espinet, \& Gonzalez, 2005). However, as the research with rats described previously illustrates clearly, if we want to demonstrate conceptual representations' being used in other animals' goal-directed decisionmaking systems, it is important that we test whether they can solve goal-directed problems in novel situations by generalizing from existing knowledge. Here, it is important that we note that the most fruitful approach to demonstrating certain higher cognitive functions in animals (such as chimpanzees, monkeys, and scrub jays) proved to be the use of a naturalistic setting, thus tapping into natural behaviors. For the purposes of probing conceptual representations, we need to be clear that a generalization or transfer task cannot be one that is solved through previously learned, habit-based behavioral responses.

At this stage, our claim is that it is a live hypothesis that the goal-directed decision-making systems in other animals are restricted to nonconceptual representations - one that calls for experimental testing. In contrast, it is very plausible that humans can transcend this kind of limitation, at least sometimes. If we do, that would be evidence for conceptual representations in decision making. Of course, we hardly need more evidence that humans possess and sometimes reason with structured representations. Verbal reasoning by talking aloud is one obvious example. In contrast, although animals (in particular-but not exclusively - birds) can learn to recognize certain structures in language and could have some language-like capacities (Gentner, Fenn, Margoliash, \& Nusbaum, 2006; Toro, Trobalon, \& Sebastián-Gallés, 2005), and although great apes show some similarity to humans in the anatomy of language-like areas (Cantalupo \& Hopkins, 2001), there is thought to be little chance that these language-related structures and capacities extend to a structured representation as adaptable, flexible, and complex as is needed for human language. With respect to humans, the more interesting question is whether such structured representations are found in the operation of the goal-directed decisionmaking system. Our first-person experience of deliberate decision making strongly suggests that it is worth looking further. As we shall see in the next section, there is some neural evidence that syntactic or grammatical mechanisms are sometimes relied on in goal-directed decision making.

\section{Testing Whether Human Goal-Directed Decision Making Uses Conceptual Representations}

Our suggestion will be that neural evidence can be used to test the hypothesis that human goal-directed decision making deploys conceptual representations. The clearest case of deploying conceptual representations is in speaking and understanding natural language - in particular, in parsing the grammar of natural language. So the obvious place to look is for differential involvement of Broca's area (of the left inferior frontal gyrus) in decision-making tasks,

controlling for potential linguistic confounds in the task. Recent DTI analysis and TMS studies suggest that two fronto-temporal networks involved in language processing should be differentiated: a dorsal network that is specialized for phonological processing, connecting areas of the fronto-parietal junction with dorsal and posterior areas of the left inferior frontal gyrus; and a ventral network that is specialized for semantic processing, connecting categoryspecific representations in the temporal pole with ventral and anterior areas of the left inferior frontal gyrus (Devlin, Matthews, \& Rushworth, 2003; Devlin, Rayley, et al., 2003; Devlin et al., 2006). This division suggests that the capacity of the inferior frontal gyrus to encode or enable structured representations can be deployed in more than one domain - in the present article, both to the compositional structure of phonetics (roughly, the way sounds can be recombined to make different words) and to the compositional structure of semantics (roughly, the way words can be recombined to make different sentences, where the words carry their meaning to the new sentences-i.e., the way concepts can be recombined to make different thoughts). Our hypothesis is that the same capacity can be deployed by the goal-directed decision-making system when reasoning with information about the relations of cause and effect between events in the world and between its own actions and those events.

Koechlin and Jubault (2006) uncovered evidence that can be deployed in support of our hypothesis (differing somewhat from their own preferred interpretation of the data). They started with the observation that actions can be chained together into sequences to form an action chunk. A sung melody is an action chunk. So is a learned sequence of buttonpresses, like LRLLRLLRLR. Once learned, an action chunk is available for use, as and when it is required. Contrast an action chunk with a complex rule for action. For example, in sorting a deck of cards, I might have the rule that red cards go $\mathrm{L}$ and black cards go $\mathrm{R}$. The rule is complex, but the temporal sequence of each action chunk is simple, involving just one movement (if I see red, I go L) before the end of the action sequence. Just as individual actions can be chained into sequences, so can complex rules for action. I might learn three ways to divide up a deck of cards: by color, by whether there is a picture card, and by even/odd numbers (counting Queen as even). I can deploy these rules in sequence: color $\rightarrow$ picture $\rightarrow$ parity $\rightarrow$ color $\rightarrow$ color $\rightarrow$ picture $\rightarrow$ picture $\rightarrow$ parity $\rightarrow$ parity. Each of these rules is itself complex, so the sequence actually goes as follows:

A1:

$\{$ red $\rightarrow \mathrm{L}\} \rightarrow$ picture $\rightarrow \mathrm{L}$ \}

$\{$ black $\rightarrow \mathrm{R}$ \}

Koechlin and Jobault reported bilateral fMRI activation in the inferior frontal gyrus (IFG) that was associated with subjects' performing the task requiring switching between task sets, as compared with a task with the same temporal complexity but lacking the superordinate structure. They inferred that hierarchical processing is different from temporal organization of goal-directed behavior, which is encoded in the lateral prefrontal cortex in a forward-moving 
cascade as the tasks become more temporally complex (Koechlin, Ody, \& Kouneiher, 2003; although the frontal pole activation may instead reflect a mechanism for keeping an alternative option for action online while making a choice [Rushworth, Behrens, Rudebeck, \& Walton, 2007]). What differentiates the task that activates IFG-according to Koechlin and Jubault - is its hierarchical structure. It chains between a series of action types-A1, A2, and A3but each is itself a complex sensorimotor mapping, making the series "hierarchical" in a way that a series of straightforwardly noncontingent actions (e.g., LRLLR) is not. An alternative but related interpretation of their results is that they are finding the signature of conceptual representation, and that such representations are deployed preferentially in the hierarchical condition. Our interpretation relates to that of Koechlin and Jobault, because the representation of task hierarchy that they identified is plausibly one with constituent structure - that is, conceptual. To chain together a series of basic actions - for example, a series of left and right buttonpresses - does not seem to require any of those actions to be generated by representations with constituent structure. It would be enough to chain through a series of nonconceptual representations that were suitably linked to the project. Our hypothesis is that purely nonconceptual representations would be inadequate to the task of chaining through a series of complex decision rules (A1, A2, and $A 3$ ). If the chain is $A 1 \rightarrow A 2 \rightarrow A 3$, but each action type requires different responses in different situations, it is hard to resist the thought that those associations are somehow to be stored along with the chain. The chain $\mathrm{A} 1 \rightarrow \mathrm{A} 2 \rightarrow \mathrm{A} 3$ is then properly understood as a chain between a series of mental representations, each with conceptual structure. Koechlin and Jubault's results, then, are consistent with the IFG activation they find being a signature of the deployment by their subjects of conceptual representations in one type of case, but not in the other.

The obvious follow-up question to ask is whether human goal-directed decision making always makes use of conceptual representations, or whether it is only sometimes that conceptual representations are involved. Koechlin and Jubault's (2006) results suggest that selecting actions in a simple action chunk (e.g., LRLLR) does not place any reliance on syntactic processing areas. However, much more work is clearly needed on whether and how extensively human goal-directed decision making really does make use of brain mechanisms that process constituent or syntactic structure. Most ambitiously, it may be possible to construct a type of task that is susceptible to either strategy and to use some kind of task priming to encourage subjects to navigate the task in either a conceptual or concept-free way. We would predict more IFG activation when subjects perform the task relying on conceptual representations (Koechlin \& Jubault's [2006] results suggest bilateral activation, unlike the left-lateralized activation more common in language tasks). In such tasks, we would also expect to find the behavioral indications of conceptbased decision making mentioned in Section 3: that one is inclined to make wider generalizations in the first place, and, in learning, is inclined to transfer the feedback from experience to a wider range of related tasks. This suggests a further prediction, if humans do indeed sometimes use both conceptual mechanisms and concept-free mechanisms in goal-directed decision making. The prediction is that neural data should predict patterns of transfer: Where we do find significant differences in IFG activation during learning, we should expect the results of that learning to be transferred to a wider class of cases. Finally, we can ask whether humans have the kind of limitation in their ability to display sensitivity to reward revaluation that is found in rats, as was discussed in Section 4. Our prediction is that humans should show a similar limitation when they are primed to deploy only nonconceptual representational mechanisms and when syntactic-processing areas in IFG are not active, and that they should not be so limited when they deploy a conceptual representational mechanism, activating the syntactic-processing powers of areas of IFG.

\section{Conclusion}

Emerging evidence suggests that the long-established distinction between habit-based and goal-directed decision-making mechanisms can also be sustained in humans. Although the habit-based system has been extensively studied in humans, the goal-directed system is less well characterized. This review brings to that task the distinction between conceptual and nonconceptual representational mechanisms. We argue that one fruitful way of studying human goal-directed decision making is to investigate the extent to which it deploys conceptual representations.

\section{AUTHOR NOTE}

The collaboration between the authors was made possible by a grant from the Volkswagen foundation, and the article was written as part of the "European Platform for Life Sciences, Mind Sciences, and the Humanities" initiative of the Volkswagen foundation. The authors thank their other colleagues in the decision-making group of the European Platform for helpful discussion of these issues. N.S. thanks Matthew Rushworth and his research group for helping him on his quest into the empirical literature. Address correspondence to N. Shea, University of Oxford, Faculty of Philosophy, 10 Merton St., Oxford, OX1 4JJ, England (e-mail: nicholas.shea@philosophy.ox.ac.uk).

\section{REFERENCES}

Adams, C. D., \& Dickinson A. (1981). Actions and habits: Variations in associative representations during instrumental learning. In N. E. Spear \& R. R. Miller (Eds.), Information processing in animals: Memory mechanisms (pp. 143-166). Hillsdale, NJ: Erlbaum.

Allen, C., \& Hauser, M. (1996). Concept attribution in nonhuman animals: Theoretical and methodological problems in ascribing complex mental processes. In M. Bekoff \& D. Jamieson (Eds.), Readings in animal cognition (pp. 47-62). Cambridge, MA: MIT Press.

Balleine, B. W., \& Dickinson, A. (1998a). Goal-directed instrumental action: Contingency and incentive learning and their cortical substrates. Neuropharmacology, 37, 407-419.

Balleine, B. W., \& Dickinson, A. (1998b). The role of incentive learning in instrumental outcome revaluation by sensory-specific satiety. Animal Learning \& Behavior, 26, 46-59.

Balleine, B. W., Espinet, A., \& Gonzalez, F. (2005). Perceptual learning enhances retrospective revaluation of conditioned flavor preferences in rats. Journal of Experimental Psychology: Animal Behavior Processes, 31, 341-350.

Baxter, M. G., Parker, A., Lindner, C. C., Izquierdo, A. D., \& MurRAY, E. A. (2000). Control of response selection by reinforcer value requires interaction of amygdala and orbital prefrontal cortex. Journal of Neuroscience, 20, 4311-4319. 
Behrens, T. E., Woolrich, M. W., Walton, M. E., \& RushWORTH, M. F. S. (2007). Learning the value of information in an uncertain world. Nature Neuroscience, 10, 1214-1221.

Bermúdez, J. (2003). Thinking without words. Cambridge, MA: MIT Press.

BERmúdeZ, J. (2006). Animal reasoning and proto-logic. In S. Hurley \& M. Nudds (Eds.), Rational animals? (pp. 127-138). Oxford: Oxford University Press.

BERRIDGE, K. C. (2001). Reward learning: Reinforcement, incentives and expectations. In D. L. Medin (Ed.), The psychology of learning and motivation (Vol. 41, pp. 223-278). San Diego: Academic Press.

Birrell, J. M., \& Brown, V. J. (2000). Medial frontal cortex mediates perceptual attentional set shifting in the rat. Journal of Neuroscience, 20, 4320-4324.

BlodgetT, H. C. (1929). The effect of the introduction of reward upon the maze performance of rats. University of California Publications in Psychology, 4, 113-134.

Cantalupo, C., \& Hopkins, W. D. (2001). Asymmetric Broca's area in great apes. Nature, 414, 505 .

Chater, N., \& Heyes, C. (1994). Animal concepts: Content and discontent. Mind \& Language, 9, 209-246.

Clayton, N. S., \& Dickinson, A. (1998). Episodic-like memory during cache recovery by scrub jays. Nature, 395, 272-274.

Coutureau, E., \& Killcross, S. (2003). Inactivation of the infralimbic prefrontal cortex reinstates goal-directed responding in overtrained rats. Behavioral \& Brain Research, 146, 167-174.

Critchley, H. D., \& Rolls, E. T. (1996). Hunger and satiety modify the responses of olfactory and visual neurons in the primate orbitofrontal cortex. Journal of Neurophysiology, 75, 1673-1686.

DaW, N. D., Niv, Y., \& DaYAN, P. (2005). Uncertainty-based competition between prefrontal and dorsolateral striatal systems for behavioral control. Nature Neuroscience, 8, 1704-1711.

Devlin, J. T., Matthews, P. M., \& Rushworth, M. F. (2003). Semantic processing in the left inferior prefrontal cortex: A combined functional magnetic resonance imaging and transcranial magnetic stimulation study. Journal of Cognitive Neuroscience, 15, 71-84.

Devlin, J. T., Raley, J., Tunbridge, E., Lanary, K., Floyer-Lea, A., NARAIN, C., ET AL. (2003). Functional asymmetry for auditory processing in human primary auditory cortex. Journal of Neuroscience, 23, 11516-11522.

Devlin, J. T., Sillery, E. L., Hall, D. A., Hobden, P., Behrens, T. E., $\&$ NunEs, R. G. (2006). Reliable identification of the auditory thalamus using multi-modal structural analyses. Neurolmage, 30, 11121120 .

Dickinson, A. (1980). Contemporary animal learning theories. Cambridge: Cambridge University Press.

Dickinson, A., \& BALleine, B. (1994). Motivational control of goaldirected action. Animal Learning \& Behavior, 22, 1-18.

Dickinson, A., \& Balleine, B. (2002). The role of learning in motivation. In C. R. Gallistel (Ed.), Learning, motivation, and emotion (pp. 497-533). New York: Wiley.

Dickinson, A., Balleine, B., Watt, A., Gonzalez, F., \& Boakes, R. A. (1995). Motivational control after extended instrumental training. Animal Learning \& Behavior, 23, 197-206.

EMERY, N. J., \& CLAYTON, N. S. (2001). Effects of experience and social context on prospective caching strategies by scrub jays. Nature, 414, 443-446.

Evans, G. (1982). The varieties of reference. Oxford: Oxford University Press.

Freedman, D. J., \& Assad, J. A. (2006). Experience-dependent representation of visual categories in parietal cortex. Nature, 443, 85-88.

Freedman, D. J., Riesenhuber, M., Poggio, T., \& Miller, E. K. (2001). Categorical representation of visual stimuli in the primate prefrontal cortex. Science, 291, 260-263.

Fuster, J. M. (2000). Executive frontal functions. Experimental Brain Research, 133, 66-70.

Gentner, T. Q., Fenn, K. M., Margoliash, D., \& Nusbaum, H. C. (2006). Recursive syntactic pattern learning by songbirds. Nature, 440, 1204-1207.

Gottfried, J. A., O’Doherty, J., \& Dolan, R. J. (2003). Encoding predictive reward value in human amygdala and orbitofrontal cortex. Science, 301, 1104-1107.

Hare, B., Call, J., Agnetta, B., \& Tomasello, M. (2000). Chimpan- zees know what conspecifics do and do not see. Animal Behavior, 59, 771-785.

Hare, B., Call, J., \& Tomasello, M. (2001). Do chimpanzees know what conspecifics know? Animal Behavior, 61, 139-151.

Holland, P. C., \& Gallagher, M. (2004). Amygdala-frontal interactions and reward expectancy. Current Opinion in Neurobiology, 14, 148-155.

Houk, J. C., Adams, J. L., \& Barto, A. G. (1995). A model of how the basal ganglia generate and use neural signals that predict reinforcement. In J. C. Houk, J. L. Davis, \& D. G. Beiser (Eds.), Models of information processing in the basal ganglia (pp. 249-270). Boston: MIT Press.

Hurley, S., \& Nudds, M. (Eds.). (2006). Rational animals? Oxford: Oxford University Press.

Jog, M. S., Kubota, Y., Connolly, C. I., Hillegaart, V., \& GrayBIEL, A. M. (1999). Building neural representations of habits. Science, 286, 1745-1749.

Johnson, A., \& Redish, A. D. (2007). Neural ensembles in CA3 transiently encode paths forward of the animal at a decision point. Journal of Neuroscience, 27, 12176-12189.

Kennerley, S. W., Walton, M. E., Behrens, T. E. J., Buckley, M. J., \& Rushworth, M. F. S. (2006). Optimal decision making and the anterior cingulate cortex. Nature Neurosicence, 9, 940-947.

Kenward, B., Weir, A. A., Rutz, C., \& KacelniK, A. (2005). Behavioural ecology: Tool manufacture by naive juvenile crows. Nature, 433, 121.

Killcross, S., \& Coutureau, E. (2003). Coordination of actions and habits in the medial prefrontal cortex of rats. Cerebral Cortex, 13, 400-408.

Koechlin, E., \& Jubault, T. (2006). Broca's area and the hierarchical organization of human behavior. Neuron, 50, 963-974.

Koechlin, E., Ody, C., \& Kounelher, F. (2003). The architecture of cognitive control in the human prefrontal cortex. Science, 302, 1181-1185.

LaU, B., \& Glimcher, P. W. (2007). Action and outcome encoding in the primate caudate nucleus. Journal of Neuroscience, 27, 14502-14514.

McClure, S. M., Laibson, D. I., Loewenstein, G., \& Cohen, J. D. (2004). Separate neural systems value immediate and delayed monetary rewards. Science, 306, 502-507.

McGaughy J., Ross, R. S., \& Eichenbaum, H. (2008). Noradrenergic, but not cholinergic, deafferentation of prefrontal cortex impairs attentional set-shifting. Neuroscience, 153, 63-71.

McLaren, I. P. L., \& Mackintosh, N. J. (2002). Associative learning and elemental representation: II. Generalization and discrimination. Animal Learning \& Behavior, 30, 177-200.

Millikan, R. G. (2000). On clear and confused ideas. Cambridge: Cambridge University Press.

Montague, P. R., Dayan, P., \& Sejnowski, T. J. (1996). A framework for mesencephalic dopamine systems based on predictive Hebbian learning. Journal of Neuroscience, 16, 1936-1947.

MurPhy, G. L. (2004). The big book of concepts. Cambridge, MA: MIT Press.

Newsome, W. T., Britten, K. H., \& Movshon, J. A. (1989). Neuronal correlates of a perceptual decision. Nature, 341, 52-54.

O’Doherty, J., Dayan, P., Friston, K. J., Critchley, H., \& Dolan, R. J. (2003). Temporal difference models and reward-related learning in the human brain. Neuron, 38, 150-152.

O'Doherty, J., Dayan, P., Schultz, J., Deichmann, R., Friston, K., \& Dolan, R. (2004). Dissociable roles of ventral and dorsal striatum in instrumental conditioning. Science, 304, 452-454.

Ottoni, E. B., DE Resende, B. D., \& Izar, P. (2005). Watching the best nutcrackers: What capuchin monkeys (Cebus apella) know about others' tool-using skills. Animal Cognition, 8, 215-219.

PaCKard, M. G., \& Knowlton, B. J. (2002). Learning and memory functions of the basal ganglia. Annual Review of Neuroscience, 25, 563-593.

PadoA-Schioppa, C., \& Assad, J. A. (2008). The representation of economic value in the orbitofrontal cortex is invariant for changes of menu. Nature Neuroscience, 11, 95-102.

Pasupathy, A., \& Miller, E. K. (2005). Different time courses of learning-related activity in the prefrontal cortex and striatum. Nature, 433, 873-876

PeAcocke, C. (1992). A study of concepts. Cambridge, MA: MIT Press. 
Preuss, T. M. (1995). Do rats have prefrontal cortex? The Rose-WoolseyAkert program reconsidered. Journal of Cognitive Neuroscience, 7 , $1-24$.

Price, J. L. (2007). Definition of the orbital cortex in relation to specific connections with limbic and visceral structures and other cortical regions. In G. Schoenbaum, J. A. Gottfried, E. A. Murray, \& S. J. Ramus (Eds.), Linking affect to action: Critical contributions of the orbitofrontal cortex (Annals of the New York Academy of Sciences, Vol. 1121, pp. 54-71). New York: New York Academy of Sciences.

RAMIREZ, D. R., \& SAVAGE, L. M. (2007). Differential involvement of the basolateral amygdala, orbitofrontal cortex, and nucleus accumbens core in the acquisition and use of reward expectancies. Behavioral Neuroscience, 121, 896-906.

Rolls, E. T., Sienkiewicz, Z. J., \& Yaxley, S. (1989). Hunger modulates the responses to gustatory stimuli of single neurons in the caudolateral orbitofrontal cortex of the macaque monkey. European Journal of Neuroscience, 1, 53-60.

Rushworth, M. F., Behrens, T. E., Rudebeck, P. H., \& WalTON, M. E. (2007). Contrasting roles for cingulate and orbitofrontal cortex in decisions and social behavior. Trends in Cognitive Sciences, 11, 168-176.

Rustichini, A. (2008). Dual or unitary system? Two alternative models of decision making. Cognitive, Affective, \& Behavioral Neuroscience, $\mathbf{8}, 355-362$.

Saito, N., Mushiake, H., Sakamoto, K., Itoyama, Y., \& Tanji, J. (2005). Representation of immediate and final behavioral goals in the monkey prefrontal cortex during an instructed delay period. Cereberal Cortex, 15, 1535-1546.

Schultz, W., Dayan, P., \& Montague, P. R. (1997). A neural substrate of prediction and reward. Science, 275, 1593-1599.

Seymour, B., O’Doherty, J., Dayan, P., Koltzenburg, M., Jones, A. K., Dolan, R. J., ET AL. (2004). Temporal difference models describe higher-order learning in humans. Nature, 429, 664-667.

Shima, K., Isoda, M., Mushiake, H., \& TANJI, J. (2007). Categorization of behavioral sequences in the prefrontal cortex. Nature, 445, 315-318.

Shipley, B. E., \& Colwill, R. M. (1996). Direct effects on instrumental performance of outcome revaluation by drive shifts. Animal Learning \& Behavior, 24, 57-67.

STICH, S. P. (1978). Do animals have beliefs? Australasian Journal of Philosophy, 57, 15-28.

Sugrue, L. P., Corrado, G. S., \& Newsome, W. T. (2004). Matching behavior and the representation of value in the parietal cortex. Science, 304, 1782-1787.

Sugrue, L. P., Corrado, G. S., \& Newsome, W. T. (2005). Choosing the greater of two goods: Neural currencies for valuation and decision making. Nature Reviews Neuroscience, 6, 1-13.
Tanaka, S. C., Balleine, B. W., \& O’Doherty, J. P. (2008). Calculating consequences: Brain systems that encode the causal effects of actions. Journal of Neuroscience, 28, 6750-6755.

Tanji, J., Shima, K., \& Mushiake, H. (2007). Concept-based behavioral planning and the lateral preforontal cortex. Trends in Cognitive Sciences, 17, 528-534.

Tobler, P. N., O’Doherty, J. P., Dolan, R. J., \& Schultz, W. (2007). Reward value coding distinct from risk attitude-related uncertainty coding in human reward systems. Journal of Neurophysiology, 97, 1621-1632.

Toro, J. M., Trobalon, J. B., \& Sebastián-Gallés, N. (2005). Effects of backward speech and speaker variability in language discrimination by rats. Journal of Experimental Psychology: Animal Behavior Processes, 31, 95-100.

Tremblay, L., \& Schultz, W. (1999). Relative reward preference in primate orbitofrontal cortex. Nature, 398, 704-708.

VAlentin, V. V., Dickinson, A., \& O'Doherty, J. P. (2007). Determining the neural substrates of goal-directed learning in the human brain. Journal of Neuroscience, 27, 4019-4026.

Walton, M., Rudebeck, P. H., Bannerman, D., \& Rushworth, M. (2007). Calculating the cost of acting in prefrontal cortex. In B. W. Balleine, K. Doya, J. O'Doherty, \& M. Sakagami (Eds.), Reward and decision making in corticobasal ganglia networks (Annals of the New York Academy of Sciences, Vol. 1104, pp. 340-356). New York: New York Academy of Sciences.

Whiten A., Spiteri A., Horner V., Bonnie K. E., Lambeth, S. P., Schapiro, S. J., \& DE WAAL, F. B. (2007). Transmission of multiple traditions within and between chimpanzee groups. Current Biology, 17, 1038-1043.

Wright, A. A., \& Katz, J. S. (2007). Generalization hypothesis of abstract-concept learning: Learning strategies and related issues in Macaca mulatta, Cebus apella, and Columba livia. Journal of Comparative Psychology, 121, 387-397.

YANG, T., \& ShadLEN, M. N. (2007). Probabilistic reasoning by neurons. Nature, 447, 1075-1080.

Yin, H. H., Knowlton, B. J., \& Balleine, B. W. (2004). Lesions of dorsolateral striatum preserve outcome expectancy but disrupt habit formation in instrumental learning. European Journal of Neuroscience, 19, 181-189.

Yin, H. H., Knowlton, B. J., \& Balleine, B. W. (2005). Blockade of NMDA receptors in the dorsomedial striatum prevents actionoutcome learning in instrumental conditioning. European Journal of Neuroscience, 22, 505-512.

(Manuscript received March 3, 2008; revision accepted for publication August 26, 2008.) 